\title{
Therapeutic efficacy of artemether-lumefantrine combination in the treatment of uncomplicated malaria among children under 5 years in 3 ecological zones in Ghana
}

\author{
Benjamin Abuaku*, Nancy Duah, Lydia Quaye, Neils Quashie, Kwadwo Koram
}

From Challenges in malaria research

Basel, Switzerland. 10-12 October 2012

\section{Background}

In 2008 artemether-lumefantrine and dihydroartemisininpiperaquine were added to amodiaquine - artesunate as first line drugs for uncomplicated malaria in Ghana. The introduction of new drugs calls for continuous monitoring of these drugs to provide timely information on trends of their efficacy and safety to enhance timely evidence-based decision making by the National Malaria Control Programme. In this regard, we monitored the therapeutic efficacy of artemether - lumefantrine from September 2010 to April 2011 in 4 sentinel sites representing the 3 main ecological zones of the country.

\section{Materials and methods}

The study population involved all children aged between 6 and 59 months presenting at the Out-Patient Department (OPD) of a study site clinic with symptoms suggestive of malaria. Using the $2009 \mathrm{WHO}$ protocol for surveillance of antimalarial drug efficacy, primary outcomes for the study were treatment outcomes on Day 14 and Day 28 for the different ecological zones whilst secondary outcomes were patterns of fever and parasite clearance as well as gametocyte carriage and haematological responses. The Institutional Review Board of the Noguchi Memorial Institute for Medical Research, University of Ghana, reviewed and approved the study.

\section{Results}

Per-protocol analysis showed that the overall pcrcorrected cure rates on day 14 and day 28 were $96.5 \%$

Epidemiology Department, Noguchi Memorial Institute for Medical Research, College of Health Sciences, University of Ghana, P. O. Box LG581, Legon, Ghana
(95\% CI: 92.1, 98.6) and 95.4\% (95\% CI: 90.3, 98.0), respectively, with statistically significant differences between the ecological zones. The $90.4 \%$ day- 28 cure rate observed in the savannah zone (95\% CI: 78.2, 96.4) was significantly the lowest compared with $100 \%$ (95\% CI: 93.2, 99.9) in the forest zone and $93.8 \%(95 \% \mathrm{CI}: 77.8,98.9)$ in the coastal zone $(P=0.017)$. Fever and parasite clearance were slower among children enrolled in the savannah zone. The proportion of children still febrile on day 1 post-treatment was significantly highest in the savannah zone (42.9\%; 95\% CI: $30.0,56.7)$ compared with the forest zone $(16.7 \%$; $95 \%$ CI: 9.5, 27.2) and the coastal zone (10.5\%; 95\% CI: 3.4, 25.7) ( $P=0.000$ ). Additionally, $14.5 \%$ (95\% CI: 6.9, 27.2) of the children enrolled in the savannah zone were parasitaemic on day 2 post-treatment whilst no child was parasitaemic on the same day in the forest and coastal zones. Gametocytaemia after day 3 post-treatment was rare in all the zones. Mean haemoglobin concentration significantly increased only in the forest zone from $10.1 \mathrm{~g} / \mathrm{dl}(95 \% \mathrm{CI}$ : $9.6,10.5)$ on day 0 to $11.0 \mathrm{~g} / \mathrm{dl}(95 \% \mathrm{CI}: 10.6,11.4)$ on day $28(p=0.004)$.

\section{Conclusions}

We conclude that AL remains efficacious in Ghana with significant ecologic zonal differences. The savannah zone may be a potential zone for any emergence of resistant alleles as a result of the slower parasite clearance observed in the zone.

\section{Acknowledgements}

We acknowledge the contributions of Dr Constance Bart Plange (National Malaria Control Programme Manager), Dr Felicia Owusu-Antwi (WHO, Ghana), Dr Jackson Sillah (WHO-AFRO), Dr Marian Warsame (WHO, Geneva), 
and study teams. The study received financial support from the Global Fund to fight AIDS, Tuberculosis and Malaria (GFATM) and the US President's

Malaria Initiative (PMI).

Published: 15 October 2012

doi:10.1186/1475-2875-11-S1-P107

Cite this article as: Abuaku et al:: Therapeutic efficacy of artemether-

lumefantrine combination in the treatment of uncomplicated malaria

among children under 5 years in 3 ecological zones in Ghana. Malaria

Journal 2012 11(Suppl 1):P107.

Submit your next manuscript to BioMed Central and take full advantage of:

- Convenient online submission

- Thorough peer review

- No space constraints or color figure charges

- Immediate publication on acceptance

- Inclusion in PubMed, CAS, Scopus and Google Scholar

- Research which is freely available for redistribution

Submit your manuscript at www.biomedcentral.com/submit 